\title{
Quantifying the Impact of Option-Based Compensation on \\ Earnings for the 50 Largest U.S. Technology Companies
}

The Leonard N. Stern School of Business

The L. Glucksman Institute for Research in Securities Markets

Faculty Advisor: Aswath Damodaran

April 2, 2001

By Noah E. Butensky 


\section{Introduction}

This study is designed to quantify the impact of option-based compensation on net income, diluted earnings per share (diluted EPS) and operating income for the fifty largest technology companies trading on the New York Stock Exchange (NYSE) and NASDAQ. (see Appendix A for companies, industry subgroups and market caps) Specifically, it compares the net income and diluted EPS reported by each company with pro forma values, adjusted for stock option expense, as disclosed in each company's 10-K footnotes. It also estimates the impact that charging stock options as a compensation expense would have on the operating income of each company.

In addition, the study segments the data based on each company's date of initial public offering. This allows us to develop an understanding of the relative differences in the use of option-based compensation between "new economy" technology firms and "old economy" technology firms.

Finally, the study seeks to provide implications for both investors and financial accounting regulators.

\section{Acknowledgements}

In August of 2000, Bear Stearns equity research published its third annual compilation of the impact of employee stock options on earnings. Their report, entitled Accounting Issues - Employee Stock Option Expense, compares reported diluted EPS to pro forma diluted EPS for the S\&P 500, in aggregate and for each individual company.

The new study that follows is modeled on this Bear Stearns report. In large part, it borrows from the Bear Stearns report in terms of organization, structure and types of analysis. However, there are a number of major differences between this study and the Bear Stearns report. First, this study looks at a different set of companies, namely the fifty largest technology firms instead of the entire S\&P 500. Second and perhaps most importantly, this study bases most of its analysis on net income measures instead of diluted earnings per share measures. Diluted EPS measures are only used for purposes of comparing results with the Bear Stearns report. Finally, while similar to the Bear Stearns study in that it demonstrates implications for investors by looking at PE ratios, this study also adds a discussion of implications for financial accounting regulators. 


\section{Background on Option-based Compensation and Accounting Standards}

During the past twenty-five years, option-based compensation packages have become an increasingly common form of remuneration for employees at publicly traded firms. One of the reasons for this is that they help to reduce agency costs. In other words, option-based compensation aids in the alignment of management and employee interests with those of shareholders who wish to maximize stock price.

However, while the desire to reduce agency costs has helped to spread the use of option-based compensation to all industries, it is by no means the only reason for their proliferation. In fact, the largest grantors of stock-option based compensation -- technology companies -- have increased their use for a complementary but somewhat separate reason. These organizations often rely on option-based compensation for executives, as well as employees throughout the company, because they offer a cash free way to attract and retain employees. Moreover, during the dot com boom, employees were particularly excited by this form of compensation because of the significant up-side potential. Today, the number of options outstanding as a percent of outstanding stock among technology firms is more than double that of all other firms in the market (Damodaran, The Dark Side of Valuation, 2000).

With this explosion in the use of option-based compensation, it is not surprising that great debate exists among accountants and financial officers as to the best way to represent this expense in each firm's public financial statements. Prior to 1995, the accounting standard governing the recognition of expenses pertaining to employee stock options was APB No. 25, issued in 1972. Under this guideline, the cost of compensation is measured by the excess of the quoted market price over the option strike price at the time the option was granted. Since nearly all management options are granted at the money, this standard rarely resulted in the recognition of any compensation expense for the firm.

In 1995, FAS-123 was adopted to make this expense more transparent. FAS-123 offers companies two options for incorporating this expense information into their $10-\mathrm{K}$ 's. First, they can choose to report the "fair value" of options grants based on an option pricing model (e.g., the Black-Scholes model) and recognize these annually as an expense. Alternatively, companies can continue to choose the approach prescribed by APB No. 25 approach, but also report pro-forma net income and earnings per share, determined as if the fair value method of FAS-123 had been used to measure compensation cost. 
While "fair value" reporting is recommended by the FASB, only two companies in the S\&P 500 have adopted this technique, and neither of them is technology based (Bear Stearns Research, Accounting Issues - Employee Stock Option Expense, August 17, 2000). The remaining firms use the guidelines set forth in APB No. 25 and also provide pro forma information in their $10-\mathrm{K}$ footnotes. These footnotes are the primary source of data for this study.

\section{Data}

The companies selected for this study are the fifty largest technology firms trading on the NYSE and NASDAQ based on market capitalizations as of January 2, 2001. The net income and diluted EPS figures were garnered from the most recent $10-\mathrm{K}$ for each company published prior to January 2, 2001. As such, for some companies the most recent fiscal year ended in 2000, while for others it ended in 1999. The operating income information was collected from Bloomberg. In this study, the relevant values (i.e., net income, diluted EPS, operating income) are exactly as reported in the footnotes or on Bloomberg and have not been further adjusted to include or exclude continuing operations charges, non-recurring charges, or other extraordinary charges.

\section{Impact on Net Income, Diluted EPS}

Compiled in Appendix B is the reported net income and pro forma net income adjusted for stock option expense for each of the fifty largest technology companies over the last two fiscal years. Appendix B also offers the percentage difference between the reported and pro forma net income values as well as data on the year-over-year growth of each value. Similar to the Bear Stearns analysis, Appendix C compiles data on diluted earnings per share and pro forma diluted earnings per share.

\section{Aggregate differences}

In aggregate, net income for these 50 companies, declines by approximately $15.2 \%$ when the fair value of employee stock options is charged to earnings. In absolute terms, aggregate net income declines by more than $\$ 8.1$ billion from $\$ 53.2$ billion to $\$ 45.1$ billion. 
Aggregate diluted EPS shows an even greater impact. Specifically, aggregate diluted EPS declines approximately $22.8 \%$ when the fair value of employee stock options is considered as a compensation expense in accordance with FAS-123. Consistent with our premise that technology firms are amongst the largest users of option-based compensation, this percentage is significantly higher than the $6 \%$ decline found by Bear Stearns for the entire S\&P 500 (Bear Stearns Research, Accounting Issues - Employee Stock Option Expense, August 17, 2000).

While the diluted EPS values were reported in this section for comparison to the Bear Stearns study, moving forward, this study will rely on the net income measures as they are more robust. In particular, an analysis based on aggregate net income takes into account the relative size of each company's income statement, while an aggregate diluted EPS approach treats all companies, regardless of size, equally. It is likely that Bear Stearns based its analysis on aggregate diluted EPS because it is easier to calculate and more readily understood by retail investors.

\section{Percentage decline in net income}

Of the fifty companies, eleven of them exhibited pro forma net income that is more than $50 \%$ less than reported net income. For seven of them, the difference is more than $100 \%$. That is, net income goes from positive to negative for seven of the companies. The biggest loser in percentage terms is Verisign Inc. which exhibits a difference of more than 700 percent. 
Table 1: Largest Percentage Decline in Net Income

\begin{tabular}{|l|c|c|c|}
\hline Company & $\begin{array}{c}\text { Reported net } \\
\text { income (in } \\
\text { millions) }\end{array}$ & $\begin{array}{c}\text { Pro forma net } \\
\text { income } \\
\text { (in millions) }\end{array}$ & $\begin{array}{c}\text { \% Difference } \\
\text { reported vs. pro } \\
\text { forma net income }\end{array}$ \\
\hline Verisign Inc & $\$ 3.96$ & $\$(24.68)$ & $-724 \%$ \\
\hline Sycamore Networks Inc & 20.40 & $(62.34)$ & $-406 \%$ \\
\hline Broadcom Corp-CL A & 83.29 & $(105.56)$ & $-227 \%$ \\
\hline Brocade Communications Systems & 2.49 & $(1.93)$ & $-178 \%$ \\
\hline SDL Inc. & 25.21 & $(11.93)$ & $-147 \%$ \\
\hline Ciena Corp & 81.39 & $(26.24)$ & $-132 \%$ \\
\hline Vitesse Semiconductor Corp & 27.89 & $(4.00)$ & $-114 \%$ \\
\hline Network Appliance Inc & 73.79 & 3.07 & $-96 \%$ \\
\hline Rational Software Corp & 85.31 & 20.09 & $-76 \%$ \\
\hline Lucent & $1,219.00$ & 452.00 & $-63 \%$ \\
\hline Applied Micro Circuits Corp & 48.63 & 19.39 & $-60 \%$ \\
\hline
\end{tabular}

Source: Company reports

“New Economy” vs. "Old Economy” technology firms

Not surprisingly "new economy" and "old economy" technology firms do not rely on option-based compensation to the same degree. For this study, the twenty-five largest technology companies that first offered stock to the public prior to 1990 are defined as "old technology" firms, while those that have gone public since are defined as "new technology firms." Using this definition, the difference in aggregate net income for "old economy" technology firms between reported values and the pro forma values is only $10.15 \%$. In comparison, the aggregate net income of "new economy" technology firms declines more than $58 \%$ when employee stock options are charged as a compensation expense.

This significant difference between "new economy" and "old economy" technology firms is even more evident when you further segment the companies by their IPO year.

Table 2: Percentage Decrease in Net Income by Year of IPO

\begin{tabular}{|l|c|c|}
\hline Firms & Year of IPO & \% difference in net income \\
\hline New, new technology (12 firms) & $1996-$ present & $75.27 \%$ \\
\hline Old, new technology (13 firms) & $1990-1995$ & $50.35 \%$ \\
\hline New, old technology (13 firms) & $1980-1989$ & $12.89 \%$ \\
\hline Old, old technology (12 firms) & Prior to 1980 & $7.33 \%$ \\
\hline
\end{tabular}


This finding is not terribly surprising in the wake of the economic expansion of the 1990 's and the emergence of the e-economy. Numerous technology companies have gone public in recent years without positive earnings or even positive cash flow, only the promise of positive cash flows in the future. As such, these companies have relied heavily on optionbased compensation to meet expenses and grow.

\section{Growth in reported net income vs. pro forma net income}

For the fifty largest technology firms included in this study, the year-over-year aggregate growth rate in reported net income is $41.8 \%$. When the fair value compensation expense is charged to earning, this growth rate decreases to $33.2 \%$. Looking at companies individually, the impact is both positive and negative. Specifically, twenty-seven companies exhibit a decrease in their year-over-year growth rates when the fair value compensation expense is charged to earnings while twelve companies exhibit an increase. Eleven companies in the data set exhibit a decrease of more than 1,000 basis points.

Table 3: Decline in Year-over-Year Net Income Growth of More than 1,000 Basis Points

\begin{tabular}{|l|c|c|c|}
\hline Company & $\begin{array}{c}\text { Growth based on } \\
\text { reported net } \\
\text { income }\end{array}$ & $\begin{array}{c}\text { Growth based on } \\
\text { pro forma net } \\
\text { income }\end{array}$ & $\begin{array}{c}\text { Basis point } \\
\text { decline between } \\
\text { reported and pro } \\
\text { forma growth }\end{array}$ \\
\hline Broadcom Corp-CL A & $238.7 \%$ & $-1553.3 \%$ & 179,201 \\
\hline Veritas Software Corp & $-1073.8 \%$ & $-1783.6 \%$ & 70,980 \\
\hline SDL Inc. & $219.0 \%$ & $-275.7 \%$ & 49,468 \\
\hline PeopleSoft Inc & $-227.0 \%$ & $-577.9 \%$ & 35,085 \\
\hline Network Appliance Inc & $107.2 \%$ & $-74.8 \%$ & 18,201 \\
\hline Applied Micro Circuits Corp & $183.8 \%$ & $46.8 \%$ & 13,698 \\
\hline Computer Associates Intl Inc & $11.2 \%$ & $-46.1 \%$ & 5,728 \\
\hline Vitesse Semiconductor Corp & $-54.4 \%$ & $-108.3 \%$ & 5,396 \\
\hline Cisco Systems Inc. & $31.9 \%$ & $4.2 \%$ & 2,771 \\
\hline Intuit Inc & $-20.9 \%$ & $-37.4 \%$ & 1,649 \\
\hline Lucent & $-74.5 \%$ & $-89.3 \%$ & 1,479 \\
\hline
\end{tabular}

Source: Company reports

Note: Companies with negative reported earnings are excluded. 


\section{Impact on Operating Income}

The impact of option-based compensation on operating income for each of the fifty technology companies is provided in Appendix D. A marginal tax rate of $40 \%$ is used to estimate the pretax employee option-based compensation expense. The operating income is as reported by Bloomberg.

Aggregate operating income for these fifty companies decreases $18.3 \%$ when the fair value compensation expense is charged to operating earnings. Thirty-seven of the fifty companies experience a double-digit percentage decline in operating income. Nine companies actually shift from an operating gain to an operating loss.

Table 4: Companies Shifting from Operating Gain to Operating Loss (in millions)

\begin{tabular}{|l|c|c|c|}
\hline Company & $\begin{array}{c}\text { Reported } \\
\text { operating income }\end{array}$ & $\begin{array}{c}\text { Pro forma } \\
\text { operating income }\end{array}$ & $\begin{array}{c}\text { \% Change in } \\
\text { operating income }\end{array}$ \\
\hline Intuit Inc & $\$ 0.77$ & $\$(162.06)$ & $-21,46 \%$ \\
\hline Sycamore Networks Inc & 1.77 & $(136.13)$ & $-7,791 \%$ \\
\hline PeopleSoft Inc & 10.89 & $(134.96)$ & $-1,339 \%$ \\
\hline Brocade Communications & 0.85 & $(6.51)$ & $-866 \%$ \\
\hline Broadcom Corp-CL A & 143.17 & $(171.57)$ & $-220 \%$ \\
\hline BEA Systems Inc & 33.71 & $(36.51)$ & $-208 \%$ \\
\hline SDL Inc. & 33.24 & $(28.66)$ & $-186 \%$ \\
\hline Ciena Corp & 127.37 & $(52.02)$ & $-141 \%$ \\
\hline Network Appliance Inc & 105.37 & $(12.51)$ & $-112 \%$ \\
\hline
\end{tabular}

Source: Company reports, Bloomberg

\section{Pre-tax compensation expense}

In the most recent fiscal year, seven of the fifty largest technology companies crossed the $\$ 500$ million threshold for pre-tax compensation expense when the fair-value method is used. Microsoft is the leader of the group with a pro forma pre-tax compensation expense in excess of $\$ 2$ billion. 
Table 5. Compensation Expense in Excess of $\$ 500$ Million

\begin{tabular}{|l|c|}
\hline Company & $\begin{array}{r}\text { Pro Forma Pretax Stock Option Expense } \\
\text { ( in millions) }\end{array}$ \\
\hline Microsoft Corp & $\$ 2,081.67$ \\
\hline Cisco Systems Inc. & $1,865.00$ \\
\hline Lucent & $1,278.33$ \\
\hline International Business Machines & $1,080.00$ \\
\hline Oracle Corporation & 932.74 \\
\hline Intel & 756.67 \\
\hline Sun Microsystems Inc & 528.33 \\
\hline Source: Company reports, Bloomberg &
\end{tabular}

\section{Implications for Investors}

This study lends itself to a very important question. Namely, "how can investors use the pro forma information provided by each company in its $10-\mathrm{K}$ footnotes to make more informed investment decisions on individual stocks?" A quick look at the data in this report suggests that net income, operating income and growth rates might not be large as they appear. Some companies that look profitable may in fact be losing money. And while the granting of stock options has no direct impact on free cash flow, the potential exercise of these options will dilute each shareholders claim on free cash flow.

Perhaps more importantly, while pure believers in the efficient market hypothesis would argue that this pro forma "information" is already completely incorporated in current stock prices, the vast majority of investors who still look to identify undervalued and overvalued equities may be able to use this information to their advantage.

\section{Specific Example - Price Earnings Multiples}

One way investors may be able to identify market inefficiencies and under- and overvalued stocks using the pro forma data is through use of a relative value measures such as the Price-Earnings (PE) multiple. Specifically, instead of making investment decisions based on PE multiples calculated using reported net income, as many investors currently do, these decisions can also incorporate an analysis based on PE multiples using forma net income.

For example, consider an investor who values large cap technology stocks (i.e., the fifty stocks in this study) using relative PE multiples based on earnings reported in each 
company's most recent 10-K. These investors buy stocks that have a low PE multiple relative to the competitive set because they appear to be undervalued and sells companies that have high relative PE multiples because they appear to be overvalued. Moreover, the investor is more likely to invest (long and short) in those stocks with PE values further from the industry average than those near the industry average.

Of the fifty companies in the study, the investor using this decision methodology would exclude from consideration the thirteen companies with an undefined PE multiple based on either reported net income or pro forma net income. Moreover, the investor is likely to exclude from the considered set both Network Appliances and Applied Micro Circuits since their PE multiples based on pro forma net income are extreme outliers to the high end. This leaves the investor with thirty-five companies to consider. 
Table 6. Pro Forma Price Earnings Multiples

\begin{tabular}{|c|c|c|c|c|c|c|}
\hline Company & $\begin{array}{c}\text { Reported } \\
\text { PE }\end{array}$ & $\begin{array}{l}\text { Industry } \\
\text { Ranking }\end{array}$ & $\begin{array}{c}\% \text { of } \\
\text { Industry } \\
\text { Average } \\
\text { PE }\end{array}$ & \begin{tabular}{||c} 
Pro \\
Forma \\
PE
\end{tabular} & $\begin{array}{l}\text { Industry } \\
\text { Ranking }\end{array}$ & $\begin{array}{c}\% \text { of } \\
\text { Industry } \\
\text { Average } \\
\text { PE }\end{array}$ \\
\hline Pitney Bowes Inc & 12.93 & 1 & $27 \%$ & 13.28 & 1 & $20 \%$ \\
\hline Micron Technology & 13.20 & 2 & $27 \%$ & 14.85 & 2 & $22 \%$ \\
\hline Computer Associates & 15.33 & 3 & $31 \%$ & 17.55 & 4 & $26 \%$ \\
\hline First Data Corp & 16.55 & 4 & $34 \%$ & 17.40 & 3 & $26 \%$ \\
\hline Hewlett-Packard Co. & 17.13 & 5 & $40 \%$ & 17.68 & 5 & $29 \%$ \\
\hline IBM & 19.34 & 6 & $40 \%$ & 21.12 & 6 & $31 \%$ \\
\hline$\overline{X I L I N X ~ I n c}$ & 21.86 & 7 & $45 \%$ & 25.45 & 7 & $38 \%$ \\
\hline Oracle Corporation & 23.45 & 8 & $48 \%$ & 25.73 & 8 & $38 \%$ \\
\hline Intuit Inc & 24.54 & $\overline{9}$ & $50 \%$ & 36.07 & 15 & $53 \%$ \\
\hline Microsoft Corp & 24.55 & 10 & $50 \%$ & 28.30 & 10 & $42 \%$ \\
\hline Computer Sciences Corp & 24.87 & 11 & $51 \%$ & 26.27 & 9 & $39 \%$ \\
\hline KLA-Tencor Corporation & 25.01 & 12 & $51 \%$ & 32.12 & 13 & $47 \%$ \\
\hline Dell Computer Corp & 27.17 & 13 & $56 \%$ & 31.39 & 12 & $46 \%$ \\
\hline IMS Health Inc & 28.32 & 14 & $58 \%$ & 34.16 & 14 & $50 \%$ \\
\hline Intel & 28.58 & 15 & $59 \%$ & 30.47 & 11 & $45 \%$ \\
\hline Teradyne Inc & 34.13 & 16 & $70 \%$ & 42.60 & 16 & $63 \%$ \\
\hline Lucent & 37.01 & 17 & $76 \%$ & 99.81 & 29 & $147 \%$ \\
\hline Applied Materials Inc & 42.91 & 18 & $88 \%$ & 50.44 & 17 & $75 \%$ \\
\hline Sun Microsystems Inc & 44.18 & 19 & $91 \%$ & 53.29 & 20 & $79 \%$ \\
\hline Compaq Computer Corp & 44.38 & 20 & $91 \%$ & 68.80 & 26 & $102 \%$ \\
\hline Altera Corporation & 45.24 & 21 & $93 \%$ & 50.71 & 18 & $75 \%$ \\
\hline Automatic Data Processing & 46.59 & 22 & $96 \%$ & 51.41 & 19 & $76 \%$ \\
\hline Maxim Integrated Products & 46.97 & 23 & $96 \%$ & 62.41 & 23 & $92 \%$ \\
\hline Adobe Systems Inc & 47.24 & 24 & $97 \%$ & 56.50 & 21 & $83 \%$ \\
\hline Linear Tech Corp & 49.92 & 25 & $102 \%$ & 58.18 & 22 & $86 \%$ \\
\hline Texas Instruments Inc & 56.98 & 26 & $117 \%$ & 63.54 & 24 & $94 \%$ \\
\hline DST Systems Inc & 57.19 & 27 & $117 \%$ & 65.81 & 25 & $97 \%$ \\
\hline Electronic Data Systems & 61.94 & 28 & $127 \%$ & 69.30 & 27 & $102 \%$ \\
\hline Rational Software Corp & 74.74 & 29 & $153 \%$ & 317.41 & 35 & $469 \%$ \\
\hline Analog Devices & 85.40 & 30 & $175 \%$ & 103.19 & 30 & $152 \%$ \\
\hline Corning Inc. & 86.96 & 31 & $179 \%$ & 90.14 & 28 & $133 \%$ \\
\hline Cisco Systems Inc. & 89.86 & 32 & $185 \%$ & 154.78 & 32 & $229 \%$ \\
\hline EMC Corp/Mass & 117.54 & 33 & $241 \%$ & 125.75 & 31 & $186 \%$ \\
\hline PMC-Sierra Inc & 119.89 & 34 & $246 \%$ & 166.81 & 33 & $246 \%$ \\
\hline Siebel Systems Inc & 190.55 & 35 & $391 \%$ & 244.17 & 34 & $361 \%$ \\
\hline Industry Average & 48.70 & & & 67.69 & & \\
\hline
\end{tabular}

Source: Company reports

Based on reported PE, eleven companies sell at a premium to the industry average, while twenty-four companies sell at a discount. Using the pro forma PE, ten sell at a premium to the group, while twenty-five sell at a discount. Two companies move from discounts to premiums and three companies move from premiums to discounts when the investor switches from basing his investment decision on the reported PE to the pro forma PE. In many cases the premium widens relative to the rest of the industry. For example, Rational 
Software's premium widens from 1.5 times the industry average to more than 4.69 times the average. Clearly, this kind of analysis can be very valuable to the investor using relative valuation to make buy, sell, and hold decisions.

So what does all this mean to the individual investor? Perhaps, it signals a need to stay away from those firms that switch from a premium to a discount or vice versa because it is "unclear", based on these two multiples, whether the equities are under- or over- valued. Alternatively, an investor who believes that pro forma data are more accurate might take advantage of these opportunities to invest in equities that other investors who base their decisions on PE multiples would have a contrary opinion on. Finally, an investor might consider a company like Rational Software to be a clear short opportunity, as he believes it is selling at a much larger premium than the investor who bases his decision on reported data.

\section{Implications for the Financial Accounting Regulators}

This study also has important implications for financial accounting regulators. These regulators should take notice of the significant differences in income between reported and pro forma values. This recognition should push them to implement rules forcing companies to disclose more transparent information with regards to option-based compensation expenses. In particular, regulators should require companies to report this option-based information not only as part of the footnotes in their year-end $10-\mathrm{K}$ 's, but also throughout the year as companies report their unaudited quarterly earnings. Moreover, regulators should reconsider whether companies should be required to incorporate these expenses in their reported income figures, not just in the financial statement's footnotes.

\section{Conclusion}

This analysis makes clear that the impact of recording option-based compensation as a compensation expense is indeed significant. Income statement accounts such as net income, diluted EPS and operating income exhibit a difference of more than 15\%. The expense recognition approach also has a dramatic impact on historical growth rates.

As such, investors should consider these values when making investment decisions. Looking at the simple example of the PE multiple, it is easy to see the strong influence on investment decisions that incorporating pro forma based multiple analysis can have. The 
study also has implications for the financial accounting regulators who should consider revising the disclosure guidelines with respect to option-based compensation to make this information more transparent.

This study leaves open to further thought and research a number of important questions. For example, is the difference observed between "new economy" and "old economy" technology stocks actually a function of age or of an underlying variable such as size, maturity, or industry subset. Another interesting question is whether pro forma values are actually built into current stock valuations? That is, to what degree does the current market take into account the pro forma net income when pricing stocks?

By building a greater understanding of these complex questions, investors can make more informed decisions and accounting regulators can implement better rules for financial disclosure. 
Appendix A - Firms, Date of IPO, Industry Subgroup, Market Capitalization (in millions)

\begin{tabular}{|c|c|c|c|}
\hline Company & $\begin{array}{l}\text { Offering } \\
\text { Year }\end{array}$ & Industry Subgroup & $\begin{array}{c}\text { Market Cap as } \\
\text { of 1-2-01 }\end{array}$ \\
\hline Cisco Systems Inc. & 1990 & Networking products & $\$ 239,755.00$ \\
\hline Microsoft Corp & 1986 & Applications software & $231,290.10$ \\
\hline Intel & 1972 & Electronic components & $209,050.60$ \\
\hline IBM & Pre-1962 & Computers & $148,793.30$ \\
\hline Oracle Corporation & 1986 & Enterprise software & $147,632.60$ \\
\hline EMC Corp/Mass & 1988 & Computers-memory & $118,836.60$ \\
\hline Sun Microsystems Inc & 1986 & Computers & $81,908.23$ \\
\hline Texas Instruments Inc & Pre-1962 & Electronic components & $80,118.19$ \\
\hline Hewlett-Packard Co. & Pre-1962 & Computers & $59,816.05$ \\
\hline Dell Computer Corp & 1988 & Computers & $45,270.86$ \\
\hline Lucent & 1996 & Networking products & $45,115.30$ \\
\hline Corning Inc. & Pre-1962 & Telecom equipment fiber & $41,888.36$ \\
\hline JDS Uniphase Corp & 1993 & Telecom equipment fiber & $39,287.82$ \\
\hline Automatic Data Processing & 1967 & Data processing/mgmt & $39,171.16$ \\
\hline Juniper Networks Inc & 1999 & Networking products & $32,569.57$ \\
\hline Applied Materials Inc & 1972 & Semiconductor equipment & $32,039.83$ \\
\hline Veritas Software Corp & 1993 & Computers-memory & $26,970.79$ \\
\hline Electronic Data Systems & 1971 & Computer service & $26,070.40$ \\
\hline Compaq Computer Corp & 1983 & Computers & $25,251.05$ \\
\hline Siebel Systems Inc & 1996 & Applications software & $23,195.29$ \\
\hline BEA Systems Inc & 1998 & Enterprise software & $20,426.67$ \\
\hline Micron Technology & 1984 & Electronic components & $19,857.27$ \\
\hline First Data Corp & 1992 & Data processing/mgmt & $19,850.31$ \\
\hline Ciena Corp & 1997 & Telecom equipment fiber & $18,875.21$ \\
\hline Applied Micro Circuits & 1997 & Electronic components & $18,848.13$ \\
\hline Broadcom Corp-CL A & 1998 & Electronic components & $18,551.63$ \\
\hline Analog Devices & 1972 & Semiconductor Compo-In & $16,807.42$ \\
\hline Brocade Communications & 1999 & Computers-integration & $16,674.83$ \\
\hline Network Appliance Inc & 1995 & Networking products & $16,503.45$ \\
\hline Linear Tech Corp & 1986 & Semiconductor Compo-In & $14,371.17$ \\
\hline XILINX Inc & 1990 & Electronic components & $14,259.53$ \\
\hline Maxim Integrated Products & 1988 & Semiconductor Compo-In & $13,180.29$ \\
\hline Verisign Inc & 1998 & Internet Security & $12,203.23$ \\
\hline
\end{tabular}


Appendix A (cont'd) - Firms, Date of IPO, Industry Subgroup, Market Capitalization (in millions)

\begin{tabular}{|l|c|l|c|}
\hline Company & $\begin{array}{c}\text { Offering } \\
\text { Year }\end{array}$ & Industry Subgroup & $\begin{array}{c}\text { Market Cap as } \\
\text { of 1-2-01 }\end{array}$ \\
\hline SDL Inc. & 1995 & Telecom equipment fiber & $\$ 12,124.49$ \\
\hline Adobe Systems Inc & 1986 & Electronic forms & $11,232.26$ \\
\hline PMC-Sierra Inc & 1991 & Electronic components & $10,792.43$ \\
\hline Computer Associates & 1981 & Enterprise software & $10,668.24$ \\
\hline Altera Corporation & 1988 & Electronic components & $10,133.68$ \\
\hline Computer Sciences & 1964 & Computer Services & $10,019.01$ \\
\hline PeopleSoft Inc & 1992 & Enterprise software & $9,348.41$ \\
\hline Vitesse Semiconductor & 1991 & Semiconductor Compo-In & $8,507.50$ \\
\hline Sycamore Networks Inc & 1999 & Telecom equipment fiber & $8,400.58$ \\
\hline Pitney Bowes Inc & Pre-1962 & Office Automation & $8,225.98$ \\
\hline DST Systems Inc & 1995 & Computer Services & $7,897.56$ \\
\hline IMS Health Inc & 1998 & Medical Information & $7,818.62$ \\
\hline Intuit Inc & 1993 & Applications software & $7,500.59$ \\
\hline Tibco Software Inc & 1999 & Internet infrastructure software & $7,473.43$ \\
\hline Teradyne Inc & 1970 & Semiconductor equipment & $6,542.77$ \\
\hline Rational Software Corp & 1983 & Applications software & $6,376.51$ \\
\hline KLA-Tencor Corporation & 1993 & Semiconductor equipment & $6,347.35$ \\
\hline Source:Blomberg & & & \\
\hline
\end{tabular}

Source: Bloomberg 


\begin{tabular}{|c|c|c|c|c|c|c|}
\hline Company name & $\begin{array}{l}\text { Fiscal } \\
\text { Year } \\
\text { end } \\
\end{array}$ & $\begin{array}{l}\text { Reported } \\
\text { net } \\
\text { income }\end{array}$ & $\begin{array}{c}\text { Pro forma } \\
\text { net income }\end{array}$ & $\begin{array}{c}\% \\
\text { Difference } \\
\text { reported } \\
\text { vs. pro } \\
\text { forma net } \\
\text { income } \\
\end{array}$ & $\begin{array}{l}\text { Growth } \\
\text { rate } \\
\text { reported } \\
\text { net income }\end{array}$ & $\begin{array}{c}\text { Growth } \\
\text { rate pro } \\
\text { forma net } \\
\text { income } \\
\end{array}$ \\
\hline Adobe Systems Inc & $\begin{array}{l}\text { Dec-99 } \\
\text { Dec-98 }\end{array}$ & $\begin{array}{r}\$ 237.75 \\
105.14\end{array}$ & $\begin{array}{r}\$ 198.79 \\
54.44\end{array}$ & $\begin{array}{l}-16.4 \% \\
-48.2 \%\end{array}$ & $126.1 \%$ & $265.2 \%$ \\
\hline Altera Corporation & $\begin{array}{l}\text { Dec-99 } \\
\text { Dec-98 }\end{array}$ & $\begin{array}{l}223.99 \\
154.39\end{array}$ & $\begin{array}{l}199.85 \\
139.99\end{array}$ & $\begin{array}{r}-10.8 \% \\
-9.3 \%\end{array}$ & $45.1 \%$ & $42.8 \%$ \\
\hline Analog Devices & $\begin{array}{l}\text { Oct-99 } \\
\text { Oct-98 }\end{array}$ & $\begin{array}{r}196.82 \\
82.41\end{array}$ & $\begin{array}{r}162.87 \\
56.72\end{array}$ & $\begin{array}{l}-17.2 \% \\
-31.2 \%\end{array}$ & $138.8 \%$ & $187.2 \%$ \\
\hline Applied Materials Inc & $\begin{array}{l}\text { Oct-99 } \\
\text { Oct-98 }\end{array}$ & $\begin{array}{l}746.65 \\
230.90\end{array}$ & $\begin{array}{l}635.25 \\
146.09\end{array}$ & $\begin{array}{l}-14.9 \% \\
-36.7 \%\end{array}$ & $223.4 \%$ & $334.8 \%$ \\
\hline Applied Micro Circuits Corp & $\begin{array}{r}\text { Mar-00 } \\
\text { Apr-99 }\end{array}$ & $\begin{array}{l}48.63 \\
17.13\end{array}$ & $\begin{array}{l}19.39 \\
13.20\end{array}$ & $\begin{array}{l}-60.1 \% \\
-22.9 \%\end{array}$ & $183.8 \%$ & $46.8 \%$ \\
\hline Automatic Data Processing & $\begin{array}{r}\text { Jun-00 } \\
\text { Jul-99 }\end{array}$ & $\begin{array}{l}840.80 \\
696.84\end{array}$ & $\begin{array}{l}762.00 \\
638.00\end{array}$ & $\begin{array}{l}-9.4 \% \\
-8.4 \%\end{array}$ & $20.7 \%$ & $19.4 \%$ \\
\hline BEA Systems Inc & $\begin{array}{l}\text { Jan-00 } \\
\text { Jan-99 }\end{array}$ & $\begin{array}{l}(19.57) \\
(51.58)\end{array}$ & $\begin{array}{l}(61.71) \\
(86.88)\end{array}$ & $\begin{array}{l}\text { N.M. } \\
\text { N.M. }\end{array}$ & N.M. & N.M. \\
\hline Broadcom Corp-CL A & $\begin{array}{l}\text { Dec-99 } \\
\text { Dec-98 }\end{array}$ & $\begin{array}{l}83.29 \\
24.59\end{array}$ & $\begin{array}{r}(105.56) \\
7.26\end{array}$ & $\begin{array}{r}-226.7 \% \\
-70.5 \%\end{array}$ & $238.7 \%$ & $-1553.3 \%$ \\
\hline Brocade Communications & $\begin{array}{l}\text { Oct-99 } \\
\text { Oct-98 }\end{array}$ & $\begin{array}{r}2.49 \\
(15.11)\end{array}$ & $\begin{array}{r}(1.93) \\
(15.52)\end{array}$ & $\begin{array}{r}-177.8 \% \\
\text { N.M. }\end{array}$ & N.M. & N.M. \\
\hline Ciena Corp & $\begin{array}{l}\text { Oct-00 } \\
\text { Oct-99 }\end{array}$ & $\begin{array}{l}81.39 \\
(3.92)\end{array}$ & $\begin{array}{l}(26.24) \\
(40.07)\end{array}$ & $\begin{array}{r}-132.2 \% \\
\text { N.M. }\end{array}$ & N.M. & N.M. \\
\hline Cisco Systems Inc. & $\begin{array}{l}\text { Jul-00 } \\
\text { Jul-99 }\end{array}$ & $\begin{array}{l}2,668.00 \\
2,023.00\end{array}$ & $\begin{array}{l}1,549.00 \\
1,487.00\end{array}$ & $\begin{array}{l}-41.9 \% \\
-26.5 \%\end{array}$ & $31.9 \%$ & $4.2 \%$ \\
\hline Compaq Computer Corp & $\begin{array}{l}\text { Dec-99 } \\
\text { Dec-98 }\end{array}$ & $\begin{array}{r}569.00 \\
(2,743.00)\end{array}$ & $\begin{array}{r}367.00 \\
(2,854.00)\end{array}$ & $\begin{array}{r}-35.5 \% \\
\text { N.M. }\end{array}$ & N.M. & N.M. \\
\hline Computer Associates & $\begin{array}{r}\text { Mar-00 } \\
\text { Apr-99 }\end{array}$ & $\begin{array}{l}696.00 \\
626.00\end{array}$ & $\begin{array}{r}608.00 \\
1,128.00\end{array}$ & $\begin{array}{r}-12.6 \% \\
80.2 \%\end{array}$ & $11.2 \%$ & $-46.1 \%$ \\
\hline Computer Sciences Corp & $\begin{array}{r}\text { Mar-00 } \\
\text { Apr-99 }\end{array}$ & $\begin{array}{l}402.87 \\
355.50\end{array}$ & $\begin{array}{l}381.36 \\
337.59\end{array}$ & $\begin{array}{l}-5.3 \% \\
-5.0 \%\end{array}$ & $13.3 \%$ & $13.0 \%$ \\
\hline Corning Inc. & $\begin{array}{l}\text { Dec-99 } \\
\text { Dec-98 }\end{array}$ & $\begin{array}{l}481.70 \\
394.00\end{array}$ & $\begin{array}{l}464.70 \\
385.00\end{array}$ & $\begin{array}{l}-3.5 \% \\
-2.3 \%\end{array}$ & $22.3 \%$ & $20.7 \%$ \\
\hline Dell Computer Corp & $\begin{array}{l}\text { Jan-00 } \\
\text { Jan-99 }\end{array}$ & $\begin{array}{l}1,666.00 \\
1,460.00\end{array}$ & $\begin{array}{l}1,442.00 \\
1,324.00\end{array}$ & $\begin{array}{r}-13.4 \% \\
-9.3 \%\end{array}$ & $14.1 \%$ & $8.9 \%$ \\
\hline DST Systems Inc & $\begin{array}{l}\text { Dec-99 } \\
\text { Dec-98 }\end{array}$ & $\begin{array}{r}138.10 \\
71.60\end{array}$ & $\begin{array}{r}120.00 \\
58.00\end{array}$ & $\begin{array}{l}-13.1 \% \\
-19.0 \%\end{array}$ & $92.9 \%$ & $106.9 \%$ \\
\hline Electronic Data Systems & $\begin{array}{l}\text { Dec-99 } \\
\text { Dec-98 }\end{array}$ & $\begin{array}{l}420.90 \\
743.40\end{array}$ & $\begin{array}{l}376.20 \\
710.70\end{array}$ & $\begin{array}{r}-10.6 \% \\
-4.4 \%\end{array}$ & $-43.4 \%$ & $-47.1 \%$ \\
\hline
\end{tabular}


Appendix B (cont'd) - Pro Forma Impact of Employee Stock Option Expense on Net Income (in millions)

\begin{tabular}{|c|c|c|c|c|c|c|}
\hline Company name & $\begin{array}{l}\text { Fiscal } \\
\text { Year } \\
\text { end }\end{array}$ & $\begin{array}{l}\text { Reported } \\
\text { net } \\
\text { income }\end{array}$ & $\begin{array}{l}\text { Pro forma } \\
\text { net income }\end{array}$ & $\begin{array}{c}\% \\
\text { Difference } \\
\text { reported } \\
\text { vs. pro } \\
\text { forma net } \\
\text { income }\end{array}$ & $\begin{array}{l}\text { Growth } \\
\text { rate } \\
\text { reported } \\
\text { net income }\end{array}$ & $\begin{array}{c}\text { Growth } \\
\text { rate pro } \\
\text { forma net } \\
\text { income }\end{array}$ \\
\hline EMC Corp/Mass & $\begin{array}{l}\text { Dec-99 } \\
\text { Dec-98 }\end{array}$ & $\begin{array}{r}\$ 1,011.00 \\
654.00\end{array}$ & $\begin{array}{r}\$ 945.00 \\
615.00\end{array}$ & $\begin{array}{l}-6.5 \% \\
-6.0 \%\end{array}$ & $54.6 \%$ & $53.7 \%$ \\
\hline First Data Corp & $\begin{array}{l}\text { Dec-99 } \\
\text { Dec-98 }\end{array}$ & $\begin{array}{r}1,199.70 \\
465.70\end{array}$ & $\begin{array}{r}1,140.80 \\
417.60\end{array}$ & $\begin{array}{r}-4.9 \% \\
-10.3 \%\end{array}$ & $157.6 \%$ & $173.2 \%$ \\
\hline Hewlett-Packard Co. & $\begin{array}{l}\text { Oct-99 } \\
\text { Oct-98 }\end{array}$ & $\begin{array}{l}3,104.00 \\
2,678.00\end{array}$ & $\begin{array}{l}2,996.00 \\
2,646.00\end{array}$ & $\begin{array}{l}-3.5 \% \\
-1.2 \%\end{array}$ & $15.9 \%$ & $13.2 \%$ \\
\hline IMS Health Inc & $\begin{array}{l}\text { Dec-99 } \\
\text { Dec-98 }\end{array}$ & $\begin{array}{l}276.06 \\
220.56\end{array}$ & $\begin{array}{l}228.88 \\
191.41\end{array}$ & $\begin{array}{l}-17.1 \% \\
-13.2 \%\end{array}$ & $25.2 \%$ & $19.6 \%$ \\
\hline Intel & $\begin{array}{l}\text { Dec-99 } \\
\text { Dec-98 }\end{array}$ & $\begin{array}{l}7,314.00 \\
6,068.00\end{array}$ & $\begin{array}{l}6,860.00 \\
5,755.00\end{array}$ & $\begin{array}{l}-6.2 \% \\
-5.2 \%\end{array}$ & $20.5 \%$ & $19.2 \%$ \\
\hline IBM & $\begin{array}{l}\text { Dec-99 } \\
\text { Dec-98 }\end{array}$ & $\begin{array}{l}7,692.00 \\
6,308.00\end{array}$ & $\begin{array}{l}7,044.00 \\
5,985.00\end{array}$ & $\begin{array}{l}-8.4 \% \\
-5.1 \%\end{array}$ & $21.9 \%$ & $17.7 \%$ \\
\hline Intuit Inc & $\begin{array}{r}\text { Jul-00 } \\
\text { Aug-99 }\end{array}$ & $\begin{array}{l}305.66 \\
386.56\end{array}$ & $\begin{array}{l}207.97 \\
332.30\end{array}$ & $\begin{array}{l}-32.0 \% \\
-14.0 \%\end{array}$ & $-20.9 \%$ & $-37.4 \%$ \\
\hline JDS Uniphase Corp & $\begin{array}{r}\text { Jun-00 } \\
\text { Jul-99 }\end{array}$ & $\begin{array}{l}(904.70) \\
(171.10)\end{array}$ & $\begin{array}{r}(1,110.50) \\
(228.70)\end{array}$ & $\begin{array}{l}\text { N.M. } \\
\text { N.M. }\end{array}$ & N.M. & N.M. \\
\hline Juniper Networks Inc & $\begin{array}{l}\text { Dec-99 } \\
\text { Dec-98 }\end{array}$ & $\begin{array}{r}(9.03) \\
(30.97)\end{array}$ & $\begin{array}{l}(43.49) \\
(31.14)\end{array}$ & $\begin{array}{l}\text { N.M. } \\
\text { N.M. }\end{array}$ & N.M. & N.M. \\
\hline KLA-Tencor Corporation & $\begin{array}{r}\text { Jun-00 } \\
\text { Jul-99 }\end{array}$ & $\begin{array}{r}253.80 \\
39.21\end{array}$ & $\begin{array}{r}197.61 \\
5.28\end{array}$ & $\begin{array}{l}-22.1 \% \\
-86.5 \%\end{array}$ & $547.2 \%$ & $3644.0 \%$ \\
\hline Linear Tech Corp & $\begin{array}{l}\text { Jul-00 } \\
\text { Jul-99 }\end{array}$ & $\begin{array}{l}287.91 \\
194.29\end{array}$ & $\begin{array}{l}247.01 \\
166.85\end{array}$ & $\begin{array}{l}-14.2 \% \\
-14.1 \%\end{array}$ & $48.2 \%$ & $48.0 \%$ \\
\hline Lucent & $\begin{array}{l}\text { Sep-00 } \\
\text { Oct-99 }\end{array}$ & $\begin{array}{l}1,219.00 \\
4,789.00\end{array}$ & $\begin{array}{r}452.00 \\
4,239.00\end{array}$ & $\begin{array}{l}-62.9 \% \\
-11.5 \%\end{array}$ & $-74.5 \%$ & $-89.3 \%$ \\
\hline Maxim Integrated Products & $\begin{array}{l}\text { Jun-00 } \\
\text { Jun-99 }\end{array}$ & $\begin{array}{l}280.62 \\
196.12\end{array}$ & $\begin{array}{l}211.19 \\
158.09\end{array}$ & $\begin{array}{l}-24.7 \% \\
-19.4 \%\end{array}$ & $43.1 \%$ & $33.6 \%$ \\
\hline Micron Technology & $\begin{array}{r}\text { Jul-00 } \\
\text { Aug-99 }\end{array}$ & $\begin{array}{r}1,504.20 \\
(68.90)\end{array}$ & $\begin{array}{r}1,337.50 \\
(144.20)\end{array}$ & $\begin{array}{r}-11.1 \% \\
\text { N.M. }\end{array}$ & N.M. & N.M. \\
\hline Microsoft Corp & $\begin{array}{r}\text { Jun-00 } \\
\text { Jul-99 }\end{array}$ & $\begin{array}{l}9,421.00 \\
7,785.00\end{array}$ & $\begin{array}{l}8,172.00 \\
7,109.00\end{array}$ & $\begin{array}{r}-13.3 \% \\
-8.7 \%\end{array}$ & $21.0 \%$ & $15.0 \%$ \\
\hline Network Appliance Inc & $\begin{array}{l}\text { Apr-00 } \\
\text { Apr-99 }\end{array}$ & $\begin{array}{l}73.79 \\
35.61\end{array}$ & $\begin{array}{r}3.07 \\
12.16\end{array}$ & $\begin{array}{l}-95.8 \% \\
-65.8 \%\end{array}$ & $107.2 \%$ & $-74.8 \%$ \\
\hline Oracle Corporation & $\begin{array}{r}\text { May-00 } \\
\text { Jun-99 }\end{array}$ & $\begin{array}{l}6,296.80 \\
1,289.76\end{array}$ & $\begin{array}{l}5,737.16 \\
1,095.97\end{array}$ & $\begin{array}{r}-8.9 \% \\
-15.0 \%\end{array}$ & $388.2 \%$ & $423.5 \%$ \\
\hline PeopleSoft Inc & $\begin{array}{l}\text { Dec-99 } \\
\text { Dec-98 }\end{array}$ & $\begin{array}{r}(177.77) \\
139.94\end{array}$ & $\begin{array}{r}(265.27) \\
55.51\end{array}$ & $\begin{array}{r}\text { N.M. } \\
-60.3 \%\end{array}$ & $-227.0 \%$ & $-577.9 \%$ \\
\hline
\end{tabular}


Appendix B (cont'd) - Pro Forma Impact of Employee Stock Option Expense on Net Income (in millions)

\begin{tabular}{|c|c|c|c|c|c|c|}
\hline Company name & $\begin{array}{l}\text { Fiscal } \\
\text { Year } \\
\text { end } \\
\end{array}$ & $\begin{array}{l}\text { Reported } \\
\text { net } \\
\text { income }\end{array}$ & $\begin{array}{c}\text { Pro forma } \\
\text { net income }\end{array}$ & $\begin{array}{c}\% \\
\text { Difference } \\
\text { reported } \\
\text { vs. pro } \\
\text { forma net } \\
\text { income } \\
\end{array}$ & $\begin{array}{c}\text { Growth } \\
\text { rate } \\
\text { reported } \\
\text { net income }\end{array}$ & $\begin{array}{c}\text { Growth } \\
\text { rate pro } \\
\text { forma net } \\
\text { income }\end{array}$ \\
\hline Pitney Bowes Inc & $\begin{array}{l}\text { Dec-99 } \\
\text { Dec-98 }\end{array}$ & $\begin{array}{r}\$ 636.21 \\
576.39\end{array}$ & $\begin{array}{r}\$ 619.63 \\
567.91\end{array}$ & $\begin{array}{l}-2.6 \% \\
-1.5 \%\end{array}$ & $10.4 \%$ & $9.1 \%$ \\
\hline PMC-Sierra Inc & $\begin{array}{l}\text { Dec-99 } \\
\text { Dec-98 }\end{array}$ & $\begin{array}{l}90.02 \\
(5.95)\end{array}$ & $\begin{array}{r}64.70 \\
(17.50)\end{array}$ & $\begin{array}{r}-28.1 \% \\
\text { N.M. }\end{array}$ & N.M. & N.M. \\
\hline Rational Software Corp & $\begin{array}{r}\text { Mar-00 } \\
\text { Apr-99 }\end{array}$ & $\begin{array}{l}85.31 \\
59.25\end{array}$ & $\begin{array}{l}20.09 \\
13.64\end{array}$ & $\begin{array}{l}-76.5 \% \\
-77.0 \%\end{array}$ & $44.0 \%$ & $47.3 \%$ \\
\hline SDL Inc. & $\begin{array}{l}\text { Dec-99 } \\
\text { Dec-98 }\end{array}$ & $\begin{array}{r}25.21 \\
7.90\end{array}$ & $\begin{array}{r}(11.93) \\
6.79\end{array}$ & $\begin{array}{r}-147.3 \% \\
-14.1 \%\end{array}$ & $219.0 \%$ & $-275.7 \%$ \\
\hline Siebel Systems Inc & $\begin{array}{l}\text { Dec-99 } \\
\text { Dec-98 }\end{array}$ & $\begin{array}{r}121.73 \\
43.46\end{array}$ & $\begin{array}{l}95.00 \\
23.20\end{array}$ & $\begin{array}{l}-22.0 \% \\
-46.6 \%\end{array}$ & $180.1 \%$ & $309.4 \%$ \\
\hline Sun Microsystems Inc & $\begin{array}{r}\text { Jun-00 } \\
\text { Jul-99 }\end{array}$ & $\begin{array}{l}1,854.00 \\
1,030.00\end{array}$ & $\begin{array}{r}1,537.00 \\
900.00\end{array}$ & $\begin{array}{l}-17.1 \% \\
-12.6 \%\end{array}$ & $80.0 \%$ & $70.8 \%$ \\
\hline Sycamore Networks Inc & $\begin{array}{r}\text { Jul-00 } \\
\text { Aug-99 }\end{array}$ & $\begin{array}{r}20.40 \\
(19.49)\end{array}$ & $\begin{array}{l}(62.34) \\
(21.31)\end{array}$ & $\begin{array}{r}-405.6 \% \\
\text { N.M. }\end{array}$ & N.M. & N.M. \\
\hline Teradyne Inc & $\begin{array}{l}\text { Dec-99 } \\
\text { Dec-98 }\end{array}$ & $\begin{array}{l}191.69 \\
102.12\end{array}$ & $\begin{array}{r}153.60 \\
77.80\end{array}$ & $\begin{array}{l}-19.9 \% \\
-23.8 \%\end{array}$ & $87.7 \%$ & $97.4 \%$ \\
\hline Texas Instruments Inc & $\begin{array}{l}\text { Dec-99 } \\
\text { Dec-98 }\end{array}$ & $\begin{array}{r}1,406.00 \\
416.00\end{array}$ & $\begin{array}{r}1,261.00 \\
329.00\end{array}$ & $\begin{array}{l}-10.3 \% \\
-20.9 \%\end{array}$ & $238.0 \%$ & $283.3 \%$ \\
\hline Tibco Software Inc & $\begin{array}{l}\text { Nov-99 } \\
\text { Nov-98 }\end{array}$ & $\begin{array}{l}(19.48) \\
(12.95)\end{array}$ & $\begin{array}{l}(22.02) \\
(13.34)\end{array}$ & $\begin{array}{l}\text { N.M. } \\
\text { N.M. }\end{array}$ & N.M. & N.M. \\
\hline Verisign Inc & $\begin{array}{l}\text { Dec-99 } \\
\text { Dec-98 }\end{array}$ & $\begin{array}{r}3.96 \\
(19.74)\end{array}$ & $\begin{array}{l}(24.68) \\
(24.12)\end{array}$ & $\begin{array}{r}-723.9 \% \\
\text { N.M. }\end{array}$ & N.M. & N.M. \\
\hline Veritas Software Corp & $\begin{array}{l}\text { Dec-99 } \\
\text { Dec-98 }\end{array}$ & $\begin{array}{r}(502.96) \\
51.65\end{array}$ & $\begin{array}{r}(540.47) \\
32.10\end{array}$ & $\begin{array}{r}\text { N.M. } \\
-37.8 \%\end{array}$ & $-1073.8 \%$ & $-1783.6 \%$ \\
\hline Vitesse Semiconductor & $\begin{array}{l}\text { Sep-00 } \\
\text { Oct-99 }\end{array}$ & $\begin{array}{l}27.89 \\
61.15\end{array}$ & $\begin{array}{l}(4.00) \\
47.92\end{array}$ & $\begin{array}{r}-114.3 \% \\
-21.6 \%\end{array}$ & $-54.4 \%$ & $-108.3 \%$ \\
\hline XILINX Inc & $\begin{array}{l}\text { Apr-00 } \\
\text { Apr-99 }\end{array}$ & $\begin{array}{l}652.45 \\
102.59\end{array}$ & $\begin{array}{r}560.30 \\
65.20\end{array}$ & $\begin{array}{l}-14.1 \% \\
-36.4 \%\end{array}$ & $536.0 \%$ & $759.4 \%$ \\
\hline
\end{tabular}

Source: Company reports, Bloomberg

N.M. - Not Meaningful. 


\begin{tabular}{|c|c|c|c|c|c|c|}
\hline Company name & $\begin{array}{l}\text { Fiscal } \\
\text { Year } \\
\text { end }\end{array}$ & $\begin{array}{c}\text { Reported } \\
\text { diluted } \\
\text { EPS }\end{array}$ & $\begin{array}{l}\text { Pro forma } \\
\text { diluted } \\
\text { EPS }\end{array}$ & $\begin{array}{c}\% \\
\text { Difference } \\
\text { reported } \\
\text { vs. pro } \\
\text { forma EPS }\end{array}$ & $\begin{array}{l}\text { Growth } \\
\text { rate } \\
\text { reported } \\
\text { EPS } \\
\end{array}$ & $\begin{array}{c}\text { Growth } \\
\text { rate pro } \\
\text { forma EPS }\end{array}$ \\
\hline Adobe Systems Inc & $\begin{array}{l}\text { Dec-99 } \\
\text { Dec-98 }\end{array}$ & $\begin{array}{r}\$ 1.84 \\
0.77\end{array}$ & $\begin{array}{r}\$ 1.56 \\
0.41\end{array}$ & $\begin{array}{l}-15.2 \% \\
-46.8 \%\end{array}$ & $139.0 \%$ & $280.5 \%$ \\
\hline Altera Corporation & $\begin{array}{l}\text { Dec-99 } \\
\text { Dec-98 }\end{array}$ & $\begin{array}{l}1.08 \\
0.78\end{array}$ & $\begin{array}{l}0.97 \\
0.72\end{array}$ & $\begin{array}{r}-10.2 \% \\
-7.7 \%\end{array}$ & $38.5 \%$ & $34.7 \%$ \\
\hline Analog Devices & $\begin{array}{l}\text { Oct-99 } \\
\text { Oct-98 }\end{array}$ & $\begin{array}{l}1.10 \\
0.50\end{array}$ & $\begin{array}{l}0.90 \\
0.32\end{array}$ & $\begin{array}{l}-18.2 \% \\
-36.0 \%\end{array}$ & $120.0 \%$ & $181.3 \%$ \\
\hline Applied Materials Inc & $\begin{array}{l}\text { Oct-99 } \\
\text { Oct-98 }\end{array}$ & $\begin{array}{l}1.89 \\
0.61\end{array}$ & $\begin{array}{l}1.60 \\
0.39\end{array}$ & $\begin{array}{l}-15.3 \% \\
-36.1 \%\end{array}$ & $209.8 \%$ & $310.3 \%$ \\
\hline Applied Micro Circuits Corp & $\begin{array}{r}\text { Mar-00 } \\
\text { Apr-99 }\end{array}$ & $\begin{array}{l}0.41 \\
0.16\end{array}$ & $\begin{array}{l}0.16 \\
0.12\end{array}$ & $\begin{array}{l}-61.0 \% \\
-25.0 \%\end{array}$ & $156.3 \%$ & $33.3 \%$ \\
\hline Automatic Data Processing & $\begin{array}{r}\text { Jun-00 } \\
\text { Jul-99 }\end{array}$ & $\begin{array}{l}1.31 \\
1.10\end{array}$ & $\begin{array}{l}1.18 \\
1.01\end{array}$ & $\begin{array}{l}-9.9 \% \\
-8.2 \%\end{array}$ & $19.1 \%$ & $16.8 \%$ \\
\hline BEA Systems Inc & $\begin{array}{l}\text { Jan-00 } \\
\text { Jan-99 }\end{array}$ & $\begin{array}{l}(0.06) \\
(0.18)\end{array}$ & $\begin{array}{l}(0.20) \\
(0.31)\end{array}$ & $\begin{array}{l}\text { N.M. } \\
\text { N.M. }\end{array}$ & N.M. & N.M. \\
\hline Broadcom Corp-CL A & $\begin{array}{l}\text { Dec-99 } \\
\text { Dec-98 }\end{array}$ & $\begin{array}{l}0.36 \\
0.12\end{array}$ & $\begin{array}{r}(0.53) \\
0.04\end{array}$ & $\begin{array}{r}-247.2 \% \\
-66.7 \%\end{array}$ & $200.0 \%$ & $-1425.0 \%$ \\
\hline Brocade Communications & $\begin{array}{l}\text { Oct-99 } \\
\text { Oct-98 }\end{array}$ & $\begin{array}{r}0.05 \\
(2.22)\end{array}$ & $\begin{array}{l}(0.04) \\
(2.28)\end{array}$ & $\begin{array}{r}-180.0 \% \\
\text { N.M. }\end{array}$ & N.M. & N.M. \\
\hline Ciena Corp & $\begin{array}{l}\text { Oct-00 } \\
\text { Oct-99 }\end{array}$ & $\begin{array}{r}0.27 \\
(0.01)\end{array}$ & $\begin{array}{l}(0.09) \\
(0.15)\end{array}$ & $\begin{array}{r}-133.3 \% \\
\text { N.M. }\end{array}$ & N.M. & N.M. \\
\hline Cisco Systems Inc. & $\begin{array}{l}\text { Jul-00 } \\
\text { Jul-99 }\end{array}$ & $\begin{array}{l}0.36 \\
0.29\end{array}$ & $\begin{array}{l}0.21 \\
0.21\end{array}$ & $\begin{array}{l}-41.7 \% \\
-27.6 \%\end{array}$ & $24.1 \%$ & $0.0 \%$ \\
\hline Compaq Computer Corp & $\begin{array}{l}\text { Dec-99 } \\
\text { Dec-98 }\end{array}$ & $\begin{array}{r}0.34 \\
(1.71)\end{array}$ & $\begin{array}{r}0.23 \\
(1.77)\end{array}$ & $\begin{array}{r}-32.4 \% \\
\text { N.M. }\end{array}$ & N.M. & N.M. \\
\hline Computer Associates & $\begin{array}{r}\text { Mar-00 } \\
\text { Apr-99 }\end{array}$ & $\begin{array}{l}1.25 \\
1.11\end{array}$ & $\begin{array}{l}1.12 \\
2.06\end{array}$ & $\begin{array}{r}-10.4 \% \\
85.6 \%\end{array}$ & $12.6 \%$ & $-45.6 \%$ \\
\hline Computer Sciences Corp & $\begin{array}{l}\text { Mar-00 } \\
\text { Apr-99 }\end{array}$ & $\begin{array}{l}2.37 \\
2.12\end{array}$ & $\begin{array}{l}2.25 \\
2.01\end{array}$ & $\begin{array}{l}-5.1 \% \\
-5.2 \%\end{array}$ & $11.8 \%$ & $11.9 \%$ \\
\hline Corning Inc. & $\begin{array}{l}\text { Dec-99 } \\
\text { Dec-98 }\end{array}$ & $\begin{array}{l}1.93 \\
1.39\end{array}$ & $\begin{array}{l}1.86 \\
1.35\end{array}$ & $\begin{array}{l}-3.6 \% \\
-2.9 \%\end{array}$ & $38.8 \%$ & $37.8 \%$ \\
\hline Dell Computer Corp & $\begin{array}{l}\text { Jan-00 } \\
\text { Jan-99 }\end{array}$ & $\begin{array}{l}0.61 \\
0.53\end{array}$ & $\begin{array}{l}0.53 \\
0.48\end{array}$ & $\begin{array}{r}-13.1 \% \\
-9.4 \%\end{array}$ & $15.1 \%$ & $10.4 \%$ \\
\hline DST Systems Inc & $\begin{array}{l}\text { Dec-99 } \\
\text { Dec-98 }\end{array}$ & $\begin{array}{l}2.13 \\
1.11\end{array}$ & $\begin{array}{l}1.85 \\
0.90\end{array}$ & $\begin{array}{l}-13.1 \% \\
-18.9 \%\end{array}$ & $91.9 \%$ & $105.6 \%$ \\
\hline Electronic Data Systems & $\begin{array}{l}\text { Dec-99 } \\
\text { Dec-98 }\end{array}$ & $\begin{array}{l}0.85 \\
1.50\end{array}$ & $\begin{array}{l}0.76 \\
1.43\end{array}$ & $\begin{array}{r}-10.6 \% \\
-4.7 \%\end{array}$ & $-43.3 \%$ & $-46.9 \%$ \\
\hline
\end{tabular}




\begin{tabular}{|c|c|c|c|c|c|c|}
\hline Company name & $\begin{array}{l}\text { Fiscal } \\
\text { Year } \\
\text { end } \\
\end{array}$ & $\begin{array}{c}\text { Reported } \\
\text { diluted } \\
\text { EPS }\end{array}$ & $\begin{array}{l}\text { Pro forma } \\
\text { diluted } \\
\text { EPS }\end{array}$ & $\begin{array}{c}\% \\
\text { Difference } \\
\text { reported } \\
\text { vs. pro } \\
\text { forma EPS } \\
\end{array}$ & $\begin{array}{l}\text { Growth } \\
\text { rate } \\
\text { reported } \\
\text { EPS } \\
\end{array}$ & $\begin{array}{c}\text { Growth } \\
\text { rate pro } \\
\text { forma EPS }\end{array}$ \\
\hline EMC Corp/Mass & $\begin{array}{l}\text { Dec-99 } \\
\text { Dec-98 }\end{array}$ & $\begin{array}{r}\$ 0.92 \\
0.61\end{array}$ & $\begin{array}{r}\$ 0.86 \\
0.57\end{array}$ & $\begin{array}{l}-6.5 \% \\
-6.6 \%\end{array}$ & $50.8 \%$ & $50.9 \%$ \\
\hline First Data Corp & $\begin{array}{l}\text { Dec-99 } \\
\text { Dec-98 }\end{array}$ & $\begin{array}{l}2.76 \\
1.04\end{array}$ & $\begin{array}{l}2.64 \\
0.94\end{array}$ & $\begin{array}{l}-4.3 \% \\
-9.6 \%\end{array}$ & $165.4 \%$ & $180.9 \%$ \\
\hline Hewlett-Packard Co. & $\begin{array}{l}\text { Oct-99 } \\
\text { Oct-98 }\end{array}$ & $\begin{array}{l}2.97 \\
2.52\end{array}$ & $\begin{array}{l}2.85 \\
2.47\end{array}$ & $\begin{array}{l}-4.0 \% \\
-2.0 \%\end{array}$ & $17.9 \%$ & $15.4 \%$ \\
\hline IMS Health Inc & $\begin{array}{l}\text { Dec-99 } \\
\text { Dec-98 }\end{array}$ & $\begin{array}{l}0.86 \\
0.66\end{array}$ & $\begin{array}{l}0.72 \\
0.57\end{array}$ & $\begin{array}{l}-16.3 \% \\
-13.6 \%\end{array}$ & $30.3 \%$ & $26.3 \%$ \\
\hline Intel & $\begin{array}{l}\text { Dec-99 } \\
\text { Dec-98 }\end{array}$ & $\begin{array}{l}2.11 \\
1.73\end{array}$ & $\begin{array}{l}1.98 \\
1.66\end{array}$ & $\begin{array}{l}-6.2 \% \\
-4.0 \%\end{array}$ & $22.0 \%$ & $19.3 \%$ \\
\hline IBM & $\begin{array}{l}\text { Dec-99 } \\
\text { Dec-98 }\end{array}$ & $\begin{array}{l}4.12 \\
3.29\end{array}$ & $\begin{array}{l}3.78 \\
3.12\end{array}$ & $\begin{array}{l}-8.3 \% \\
-5.2 \%\end{array}$ & $25.2 \%$ & $21.2 \%$ \\
\hline Intuit Inc & $\begin{array}{r}\text { Jul-00 } \\
\text { Aug-99 }\end{array}$ & $\begin{array}{l}1.45 \\
1.93\end{array}$ & $\begin{array}{l}0.98 \\
1.66\end{array}$ & $\begin{array}{l}-32.4 \% \\
-14.0 \%\end{array}$ & $-24.9 \%$ & $-41.0 \%$ \\
\hline JDS Uniphase Corp & $\begin{array}{r}\text { Jun-00 } \\
\text { Jul-99 }\end{array}$ & $\begin{array}{l}(1.27) \\
(0.54)\end{array}$ & $\begin{array}{l}(1.56) \\
(0.72)\end{array}$ & $\begin{array}{l}\text { N.M. } \\
\text { N.M. }\end{array}$ & N.M. & N.M. \\
\hline Juniper Networks Inc & $\begin{array}{l}\text { Dec-99 } \\
\text { Dec-98 }\end{array}$ & $\begin{array}{l}(0.10) \\
(0.80)\end{array}$ & $\begin{array}{l}(0.46) \\
(0.80)\end{array}$ & $\begin{array}{l}\text { N.M. } \\
\text { N.M. }\end{array}$ & N.M. & N.M. \\
\hline KLA-Tencor Corporation & $\begin{array}{r}\text { Jun-00 } \\
\text { Jul-99 }\end{array}$ & $\begin{array}{l}1.32 \\
0.21\end{array}$ & $\begin{array}{l}1.05 \\
0.03\end{array}$ & $\begin{array}{l}-20.5 \% \\
-85.7 \%\end{array}$ & $528.6 \%$ & $3400.0 \%$ \\
\hline Linear Tech Corp & $\begin{array}{l}\text { Jul-00 } \\
\text { Jul-99 }\end{array}$ & $\begin{array}{l}0.93 \\
0.64\end{array}$ & $\begin{array}{l}0.75 \\
0.53\end{array}$ & $\begin{array}{l}-19.4 \% \\
-17.2 \%\end{array}$ & $45.3 \%$ & $41.5 \%$ \\
\hline Lucent & $\begin{array}{l}\text { Sep-00 } \\
\text { Oct-99 }\end{array}$ & $\begin{array}{l}0.37 \\
1.43\end{array}$ & $\begin{array}{l}0.13 \\
1.27\end{array}$ & $\begin{array}{l}-64.9 \% \\
-11.2 \%\end{array}$ & $-74.1 \%$ & $-89.8 \%$ \\
\hline Maxim Integrated Products & $\begin{array}{l}\text { Jun-00 } \\
\text { Jun-99 }\end{array}$ & $\begin{array}{l}0.88 \\
0.64\end{array}$ & $\begin{array}{l}0.66 \\
0.52\end{array}$ & $\begin{array}{l}-25.0 \% \\
-18.8 \%\end{array}$ & $37.5 \%$ & $26.9 \%$ \\
\hline Micron Technology & $\begin{array}{r}\text { Jul-00 } \\
\text { Aug-99 }\end{array}$ & $\begin{array}{r}2.56 \\
(0.13)\end{array}$ & $\begin{array}{r}2.28 \\
(0.28)\end{array}$ & $\begin{array}{c}-10.9 \% \\
\text { N.M. }\end{array}$ & N.M. & N.M. \\
\hline Microsoft Corp & $\begin{array}{r}\text { Jun-00 } \\
\text { Jul-99 }\end{array}$ & $\begin{array}{l}1.70 \\
1.42\end{array}$ & $\begin{array}{l}1.48 \\
1.30\end{array}$ & $\begin{array}{r}-12.9 \% \\
-8.5 \%\end{array}$ & $19.7 \%$ & $13.8 \%$ \\
\hline Network Appliance Inc & $\begin{array}{l}\text { Apr-00 } \\
\text { Apr-99 }\end{array}$ & $\begin{array}{l}0.21 \\
0.11\end{array}$ & $\begin{array}{l}0.01 \\
0.04\end{array}$ & $\begin{array}{l}-95.2 \% \\
-63.6 \%\end{array}$ & $90.9 \%$ & $-75.0 \%$ \\
\hline Oracle Corporation & $\begin{array}{r}\text { May-00 } \\
\text { Jun-99 }\end{array}$ & $\begin{array}{l}2.10 \\
0.43\end{array}$ & $\begin{array}{l}1.91 \\
0.37\end{array}$ & $\begin{array}{r}-9.0 \% \\
-14.0 \%\end{array}$ & $388.4 \%$ & $416.2 \%$ \\
\hline PeopleSoft Inc & $\begin{array}{l}\text { Dec-99 } \\
\text { Dec-98 }\end{array}$ & $\begin{array}{r}(0.67) \\
0.50\end{array}$ & $\begin{array}{r}(1.00) \\
0.20\end{array}$ & $\begin{array}{r}\text { N.M. } \\
-60.0 \%\end{array}$ & $-234.0 \%$ & $-600.0 \%$ \\
\hline
\end{tabular}




\begin{tabular}{|c|c|c|c|c|c|c|}
\hline Company name & $\begin{array}{l}\text { Fiscal } \\
\text { Year } \\
\text { end } \\
\end{array}$ & $\begin{array}{c}\text { Reported } \\
\text { diluted } \\
\text { EPS }\end{array}$ & $\begin{array}{c}\text { Pro forma } \\
\text { diluted } \\
\text { EPS }\end{array}$ & $\begin{array}{c}\% \\
\text { Difference } \\
\text { reported } \\
\text { vs. pro } \\
\text { forma EPS }\end{array}$ & $\begin{array}{l}\text { Growth } \\
\text { rate } \\
\text { reported } \\
\text { EPS } \\
\end{array}$ & $\begin{array}{c}\text { Growth } \\
\text { rate pro } \\
\text { forma EPS }\end{array}$ \\
\hline Pitney Bowes Inc & $\begin{array}{l}\text { Dec-99 } \\
\text { Dec-98 }\end{array}$ & $\begin{array}{r}\$ 2.34 \\
2.06\end{array}$ & $\begin{array}{r}\$ 2.28 \\
2.03\end{array}$ & $\begin{array}{l}-2.6 \% \\
-1.5 \%\end{array}$ & $13.6 \%$ & $12.3 \%$ \\
\hline PMC-Sierra Inc & $\begin{array}{l}\text { Dec-99 } \\
\text { Dec-98 }\end{array}$ & $\begin{array}{r}0.60 \\
(0.05)\end{array}$ & $\begin{array}{r}0.43 \\
(0.13)\end{array}$ & $\begin{array}{c}-28.3 \% \\
\text { N.M. }\end{array}$ & N.M. & N.M. \\
\hline Rational Software Corp & $\begin{array}{r}\text { Mar-00 } \\
\text { Apr-99 }\end{array}$ & $\begin{array}{l}0.89 \\
0.65\end{array}$ & $\begin{array}{l}0.21 \\
0.15\end{array}$ & $\begin{array}{l}-76.4 \% \\
-76.9 \%\end{array}$ & $36.9 \%$ & $40.0 \%$ \\
\hline SDL Inc. & $\begin{array}{l}\text { Dec-99 } \\
\text { Dec-98 }\end{array}$ & $\begin{array}{l}0.37 \\
0.13\end{array}$ & $\begin{array}{r}(0.19) \\
0.11\end{array}$ & $\begin{array}{r}-151.4 \% \\
-15.4 \%\end{array}$ & $184.6 \%$ & $-272.7 \%$ \\
\hline Siebel Systems Inc & $\begin{array}{l}\text { Dec-99 } \\
\text { Dec-98 }\end{array}$ & $\begin{array}{l}0.54 \\
0.21\end{array}$ & $\begin{array}{l}0.42 \\
0.11\end{array}$ & $\begin{array}{l}-22.2 \% \\
-47.6 \%\end{array}$ & $157.1 \%$ & $281.8 \%$ \\
\hline Sun Microsystems Inc & $\begin{array}{r}\text { Jun-00 } \\
\text { Jul-99 }\end{array}$ & $\begin{array}{l}1.10 \\
0.63\end{array}$ & $\begin{array}{l}0.92 \\
0.55\end{array}$ & $\begin{array}{l}-16.4 \% \\
-12.7 \%\end{array}$ & $74.6 \%$ & $67.3 \%$ \\
\hline Sycamore Networks Inc & $\begin{array}{r}\text { Jul-00 } \\
\text { Aug-99 }\end{array}$ & $\begin{array}{r}0.10 \\
(2.09)\end{array}$ & $\begin{array}{l}(0.41) \\
(2.29)\end{array}$ & $\begin{array}{r}-510.0 \% \\
\text { N.M. }\end{array}$ & N.M. & N.M. \\
\hline Teradyne Inc & $\begin{array}{l}\text { Dec-99 } \\
\text { Dec-98 }\end{array}$ & $\begin{array}{l}1.07 \\
0.59\end{array}$ & $\begin{array}{l}0.86 \\
0.45\end{array}$ & $\begin{array}{l}-19.6 \% \\
-23.7 \%\end{array}$ & $81.4 \%$ & $91.1 \%$ \\
\hline Texas Instruments Inc & $\begin{array}{l}\text { Dec-99 } \\
\text { Dec-98 }\end{array}$ & $\begin{array}{l}1.68 \\
0.51\end{array}$ & $\begin{array}{l}1.52 \\
0.40\end{array}$ & $\begin{array}{r}-9.5 \% \\
-21.6 \%\end{array}$ & $229.4 \%$ & $280.0 \%$ \\
\hline Tibco Software Inc & $\begin{array}{l}\text { Nov-99 } \\
\text { Nov-98 }\end{array}$ & $\begin{array}{l}(0.19) \\
(0.22)\end{array}$ & $\begin{array}{l}(0.21) \\
(0.22)\end{array}$ & $\begin{array}{l}\text { N.M. } \\
\text { N.M. }\end{array}$ & N.M. & N.M. \\
\hline Verisign Inc & $\begin{array}{l}\text { Dec-99 } \\
\text { Dec-98 }\end{array}$ & $\begin{array}{r}0.03 \\
(0.24)\end{array}$ & $\begin{array}{l}(0.25) \\
(0.29)\end{array}$ & $\begin{array}{r}-933.3 \% \\
\text { N.M. }\end{array}$ & N.M. & N.M. \\
\hline Veritas Software Corp & $\begin{array}{l}\text { Dec-99 } \\
\text { Dec-98 }\end{array}$ & $\begin{array}{r}(1.59) \\
0.22\end{array}$ & $\begin{array}{r}(1.71) \\
0.14\end{array}$ & $\begin{array}{r}\text { N.M. } \\
-36.4 \%\end{array}$ & $-822.7 \%$ & $-1321.4 \%$ \\
\hline Vitesse Semiconductor & $\begin{array}{l}\text { Sep-00 } \\
\text { Oct-99 }\end{array}$ & $\begin{array}{l}0.15 \\
0.34\end{array}$ & $\begin{array}{r}(0.02) \\
0.27\end{array}$ & $\begin{array}{r}-113.3 \% \\
-20.6 \%\end{array}$ & $-55.9 \%$ & $-107.4 \%$ \\
\hline XILINX Inc & $\begin{array}{l}\text { Apr-00 } \\
\text { Apr-99 }\end{array}$ & $\begin{array}{l}1.90 \\
0.33\end{array}$ & $\begin{array}{l}1.60 \\
0.21\end{array}$ & $\begin{array}{l}-15.8 \% \\
-36.4 \%\end{array}$ & $475.8 \%$ & $661.9 \%$ \\
\hline
\end{tabular}

Source: Company reports, Bloomberg

N.M. - Not Meaningful. 
Appendix D - Pro Forma Impact of Employee Stock Option Expense on Operating Income (in millions)

\begin{tabular}{|c|c|c|c|c|c|c|}
\hline Company name & $\begin{array}{l}\text { Fiscal } \\
\text { year } \\
\text { end }\end{array}$ & $\begin{array}{l}\text { Reported } \\
\text { operating } \\
\text { income }\end{array}$ & $\begin{array}{c}\text { After-Tax } \\
\text { FAS-123 } \\
\text { comp. } \\
\text { exp. }\end{array}$ & $\begin{array}{c}\text { Pretax } \\
\text { FAS-123 } \\
\text { comp. } \\
\text { exp. }\end{array}$ & $\begin{array}{l}\text { Pro forma } \\
\text { operating } \\
\text { income }\end{array}$ & $\begin{array}{c}\% \text { Change } \\
\text { in } \\
\text { operating } \\
\text { income }\end{array}$ \\
\hline Adobe Systems Inc & Dec-99 & $\$ 286.46$ & $\$ 38.96$ & $\$ 64.94$ & $\$ 221.52$ & $-23 \%$ \\
\hline Altera Corporation & Dec-99 & 306.02 & 24.14 & 40.24 & 265.78 & $-13 \%$ \\
\hline Analog Devices & Oct-99 & 248.06 & 33.95 & 56.58 & 191.48 & $-23 \%$ \\
\hline Applied Materials Inc & Oct-99 & 983.81 & 111.40 & 185.66 & 798.15 & $-19 \%$ \\
\hline Applied Micro Circuits & Mar-00 & 61.12 & 29.24 & 48.73 & 12.39 & $-80 \%$ \\
\hline Automatic Data Processing & Jun-00 & $1,335.10$ & 78.80 & 131.33 & $1,203.77$ & $-10 \%$ \\
\hline BEA Systems Inc & Jan-00 & 33.71 & 42.13 & 70.22 & $(36.51)$ & $-208 \%$ \\
\hline Broadcom Corp-CL A & Dec-99 & 143.17 & 188.84 & 314.74 & $(171.57)$ & $-220 \%$ \\
\hline Brocade Communications & Oct-99 & 0.85 & 4.42 & 7.36 & $(6.51)$ & $-866 \%$ \\
\hline Ciena Corp & Oct-00 & 127.37 & 107.63 & 179.39 & $(52.02)$ & $-141 \%$ \\
\hline Cisco Systems Inc. & Jul-00 & $4,608.00$ & $1,119.00$ & $1,865.00$ & $2,743.00$ & $-40 \%$ \\
\hline Compaq Computer Corp & Dec-99 & 726.00 & 202.00 & 336.67 & 389.33 & $-46 \%$ \\
\hline Computer Associates & Mar-00 & $2,724.00$ & 88.00 & 146.67 & $2,577.33$ & $-5 \%$ \\
\hline Computer Sciences Corp & Mar-00 & 693.06 & 21.52 & 35.86 & 657.20 & $-5 \%$ \\
\hline Corning Inc. & Dec-99 & 737.40 & 17.00 & 28.33 & 709.07 & $-4 \%$ \\
\hline Dell Computer Corp & Jan-00 & $2,457.00$ & 224.00 & 373.33 & $2,083.67$ & $-15 \%$ \\
\hline DST Systems Inc & Dec-99 & 199.70 & 18.10 & 30.17 & 169.53 & $-15 \%$ \\
\hline Electronic Data Systems & Dec-99 & $1,511.00$ & 44.70 & 74.50 & $1,436.50$ & $-5 \%$ \\
\hline EMC Corp/Mass & Dec-99 & $1,449.34$ & 66.00 & 110.00 & $1,339.34$ & $-8 \%$ \\
\hline First Data Corp & Dec-99 & $1,213.40$ & 58.90 & 98.17 & $1,115.23$ & $-8 \%$ \\
\hline Hewlett-Packard Co. & Oct-99 & $3,688.00$ & 108.00 & 180.00 & $3,508.00$ & $-5 \%$ \\
\hline IMS Health Inc & Dec-99 & 339.02 & 47.18 & 78.64 & 260.38 & $-23 \%$ \\
\hline Intel & Dec-99 & $10,159.00$ & 454.00 & 756.67 & $9,402.33$ & $-7 \%$ \\
\hline IBM & Dec-99 & $11,927.00$ & 648.00 & $1,080.00$ & $10,847.00$ & $-9 \%$ \\
\hline Intuit Inc & Jul-00 & 0.77 & 97.70 & 162.83 & $(162.06)$ & $-21,146 \%$ \\
\hline JDS Uniphase Corp & Jun-00 & 392.50 & 205.80 & 343.00 & 49.50 & $-87 \%$ \\
\hline Juniper Networks Inc & Dec-99 & $(14.62)$ & 34.45 & 57.42 & $(72.04)$ & N.M. \\
\hline KLA-Tencor Corporation & Jun-00 & 306.90 & 56.19 & 93.65 & 213.25 & $-31 \%$ \\
\hline Linear Tech Corp & Jul-00 & 374.40 & 40.90 & 68.16 & 306.24 & $-18 \%$ \\
\hline Lucent & Sep-00 & $3,990.00$ & 767.00 & $1,278.33$ & $2,711.67$ & $-32 \%$ \\
\hline Maxim Integrated Products & Jun-00 & 385.39 & 69.43 & 115.71 & 269.68 & $-30 \%$ \\
\hline
\end{tabular}


Appendix D (cont'd) - Pro Forma Impact of Employee Stock Option Expense on Operating Income (in millions)

\begin{tabular}{|c|c|c|c|c|c|c|}
\hline Company name & $\begin{array}{l}\text { Fiscal } \\
\text { year } \\
\text { end }\end{array}$ & $\begin{array}{l}\text { Reported } \\
\text { operating } \\
\text { income }\end{array}$ & $\begin{array}{c}\text { After-Tax } \\
\text { FAS-123 } \\
\text { comp. } \\
\text { exp. } \\
\end{array}$ & $\begin{array}{l}\text { Pretax } \\
\text { FAS-123 } \\
\text { comp. } \\
\text { exp. }\end{array}$ & $\begin{array}{c}\text { Pro forma } \\
\text { operating } \\
\text { income }\end{array}$ & $\begin{array}{c}\% \text { Change } \\
\text { in } \\
\text { operating } \\
\text { income }\end{array}$ \\
\hline Micron Technology & Jul-00 & $2,285.20$ & 166.70 & 277.83 & $2,007.37$ & $-12 \%$ \\
\hline Microsoft Corp & Jun-00 & $10,937.00$ & $1,249.00$ & $2,081.67$ & $8,855.33$ & $-19 \%$ \\
\hline Network Appliance Inc & Apr-00 & 105.37 & 70.73 & 117.88 & $(12.51)$ & $-112 \%$ \\
\hline Oracle Corporation & May-00 & $3,080.16$ & 559.64 & 932.74 & $2,147.42$ & $-30 \%$ \\
\hline PeopleSoft Inc & Dec-99 & 10.89 & 87.51 & 145.85 & $(134.96)$ & $-1339 \%$ \\
\hline Pitney Bowes Inc & Dec-99 & $1,114.32$ & 16.59 & 27.65 & $1,086.68$ & $-2 \%$ \\
\hline PMC-Sierra Inc & Dec-99 & 98.46 & 25.32 & 42.20 & 56.26 & $-43 \%$ \\
\hline Rational Software Corp & Mar-00 & 111.67 & 65.23 & 108.71 & 2.96 & $-97 \%$ \\
\hline SDL Inc. & Dec-99 & 33.24 & 37.14 & 61.90 & $(28.66)$ & $-186 \%$ \\
\hline Siebel Systems Inc & Dec-99 & 182.95 & 26.73 & 44.55 & 138.40 & $-24 \%$ \\
\hline Sun Microsystems Inc & Jun-00 & $2,405.00$ & 317.00 & 528.33 & $1,876.67$ & $-22 \%$ \\
\hline Sycamore Networks Inc & Jul-00 & 1.77 & 82.74 & 137.90 & $(136.13)$ & $-7791 \%$ \\
\hline Teradyne Inc & Dec-99 & 258.20 & 38.09 & 63.49 & 194.71 & $-25 \%$ \\
\hline Texas Instruments Inc & Dec-99 & $1,696.00$ & 145.00 & 241.67 & $1,454.33$ & $-14 \%$ \\
\hline Tibco Software Inc & Nov-99 & $(9.01)$ & 2.54 & 4.24 & $(13.25)$ & N.M. \\
\hline Verisign Inc & Dec-99 & (3.31) & 28.63 & 47.72 & $(51.03)$ & N.M. \\
\hline Veritas Software Corp & Dec-99 & (324.38) & 37.52 & 62.53 & $(386.91)$ & N.M. \\
\hline Vitesse Semiconductor & Sep-00 & 153.58 & 31.89 & 53.15 & 100.43 & $-35 \%$ \\
\hline XILINX Inc & Apr-00 & 326.75 & 92.15 & 153.58 & 173.17 & $-47 \%$ \\
\hline
\end{tabular}

(1) Calculated using an estimated tax rate of $40 \%$. 